\title{
Effect of Plyometric Exercise on Shoulder Internal Rotation after Overuse Injuries in Adolescent
}

\author{
Mahmoud Megahed Bagago*, Khaled Ahmed Olama, Mohamed Ismail Elassal \\ Department of Physical Therapy for Pediatrics, Faculty of Physical Therapy, Cairo University, Egypt \\ *Corresponding author: Mahmoud Megahed Bagago, Mobile: (+20) 01092193365, E-Mail: mahmoudalibagago@ gmail.com
}

\begin{abstract}
Background: Shoulder overuse injuries represent a common complaint among adolescents. Overuse injuries usually occur as a result of muscles weakness and imbalance between shoulder external (ER) and internal rotation (IR). Muscle tendon units may have elevated risk of overuse injuries in the actively growing child because as the bone lengthens, the muscle-tendons have to stretch to keep up. This relative tightness and related poor flexibility place young athletes at increased risk of muscle-tendon strains, avulsion injuries, and muscle tears.

Objective: The aim of this study was to determine the effect of plyometric exercise on shoulder internal rotation for adolescent after overuse injuries.

Patients and methods: A total 30 adolescents boy children were enrolled in this study, their age ranged between 15 to 18 years old. The shoulder internal rotation was evaluated by using universal goniometer. The pain intensity was evaluated by using Wong Backer Faces pain scale (WBFS).

Results: All children were graded as 4 "hurts little" by WBFS. There was a significant decrease in glenohumeral internal rotation deficit $(\mathrm{GIRD})$ and increase shoulder internal rotation post treatment compared with that pretreatment in the group A and $\mathrm{B}(\mathrm{p}<0.001)$.

Conclusion: According to results we concluded that there was significant improvement in shoulder internal rotation and decrease in GIRD for adolescents' shoulders after overuse injuries.

Keywords: Internal rotation, Glenohumeral internal rotation deficit, Overuse injuries, Plyometrics exercise.
\end{abstract}

\section{INTRODUCTION}

Overuse injury is a broad term used to describe an injury caused by repeated micro-trauma, rather than a specific or single injury event ${ }^{[1]}$.

Muscle tendon units may have elevated risk of overuse injuries in the actively growing child because as the bone lengthens, the muscle-tendons have to stretch to keep up. This relative tightness and related poor flexibility place young athletes at increased risk of muscle-tendon strains, avulsion injuries, and muscle tears ${ }^{[2]}$.

Although little research has identified causative factors for overuse injuries in children and adolescents, these injuries may be caused by training errors, improper technique, excessive sports training, inadequate rest, muscle weakness and imbalances ${ }^{[3]}$. Overuse injuries in the pediatric population represent a significant health care concern. Some reports and clinical observations indicate that $50 \%$ of pediatric patients present to sports medicine clinics for chronic injuries ${ }^{[4]}$.

Given the incomplete development of their musculoskeletal systems, adolescents may be more susceptible to sports injuries than adult athletes. Sportspecific adaptations at the glenohumeral joint could occur during adolescence because athletes begin participating in high-performance sports in early childhood ${ }^{[5]}$.

Shoulder injuries in overhead athletes are commonly due to repetitive use muscle fatigue, may be related to scapular dyskinesia rotator cuff injury and weakness, or glenohumeral internal-rotation deficit, resulting in internal impingement or labral injury (or both) ${ }^{[6]}$.

GIRD is a reduction in the IR angle of the throwing shoulder in comparison to the non-throwing shoulder and considered a primary factor in the development of shoulder injuries ${ }^{[7]}$.

Strength balance between agonist and antagonist muscles around the scapula and shoulder provides dynamic glenohumeral joint stabilization, which is needed for optimum performance in overhead sports ${ }^{[8]}$.

Plyometric exercises are a quick, powerful movement using a pre-stretch or counter movement, which involves the stretch shortening cycle. The purpose of plyometric exercises is to increase the power of subsequent movements by using both the natural and elastic components of muscle, and tendon and the stretch reflex ${ }^{[9]}$.

The aim of this study was to determine the effect of plyometric exercise on shoulder internal rotation for adolescents after overuse injuries.

\section{PATIENTS AND METHODS}

This study included thirty male adolescents; their age ranged from 15 to 18 years old, were selected from el Menshawy General Hospital Tanta City. Those meeting the criteria of inclusion were divided according the type of intervention into two groups (group A and B). 
Group A involved 15 boys who received selected plyometric exercise plus selected traditional physical therapy program one hour per day for 3 days per week for six weeks. Group B involved 15 boys received just selected physical therapy program for 3 days per week for six weeks.

All boys were included in the analysis as they all met the inclusion criteria of being able to understand instruction and cooperate during assessment. The exclusion criteria were as follows: (1) boys undergo shoulder surgical operation; (2) previous history of fracture; (3) recurrent shoulder dislocation; (4) shoulder pain related to cervical spine; (5) boys with neurological disorders.

\section{Ethical approval:}

This experimental study was approved by the Ethical Committee of Faculty of Physical Therapy, Cairo University, Egypt and was performed in accordance with the Declaration of Helsinki of 1964 and its later amendments. All participating signed consent forms for their participation.

\section{Wong baker faces pain scale:}

Children and parents use the Wong-Baker FACES Pain Scale (WBFS) for reporting pain intensity. The Wong Baker FACES Pain Rating Scale (FACES) is a six-graphed-face pain rating scale that ranges from 0 to 10 .

Tell the person that each face represents someone who is in no pain (hurt), any pain, or a lot of pain. Face 0 is absolutely painless. Face 2 is a little bit painful. Face 4 is slightly more painful.

It's much more uncomfortable on face 6 . Face 8 was incredibly painful. Face 10 is as painful as you can think, but you don't have to be crying to experience it. Have the individual select the face that best represents their pain. All subjects selected in this study were graded level 4 hurts little more.

\section{Wong-Baker FACES "w Pain Rating Scale}
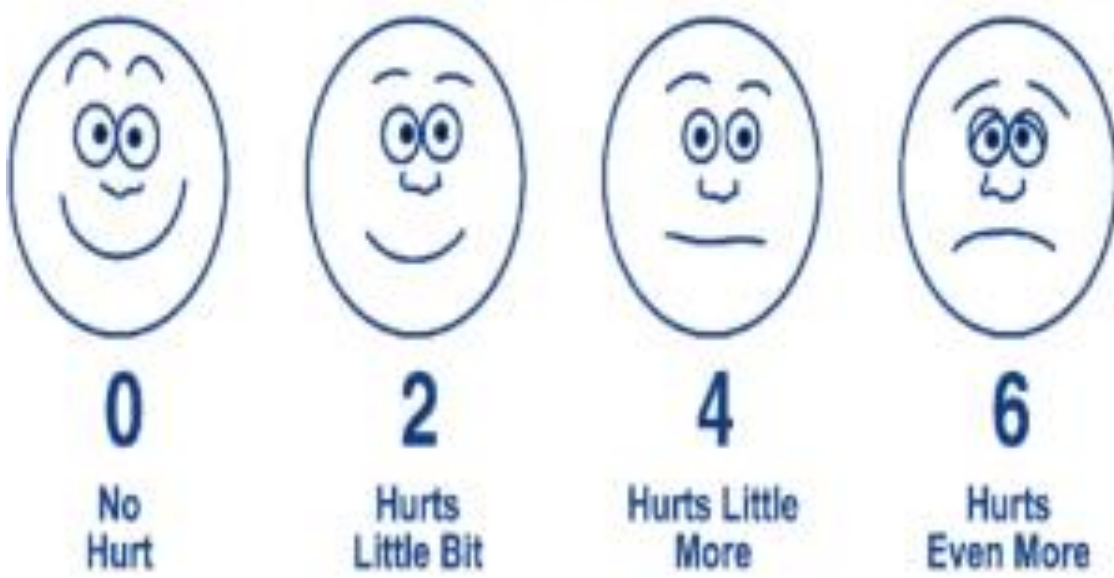

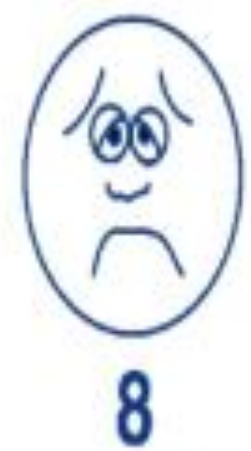

Hurts Whole Lot

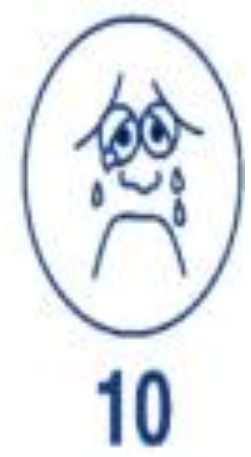

Hurts Worst

Figure (1): Wong Baker FACES pain rating scale. 


\section{Universal goniometer:}

Goniometry is used by physical therapists to assess passive and active motion range (ROM).

The measurements are often used to determine ROM limits, choose appropriate treatments, and monitor treatment progress. Each patient for each group were been measured use universal goniometer for evaluation of inward and outward rotation for both shoulders and then calculated glenohumeral internal rotation deficit GIRD before and after treatment procedure in the following sequence:

The participants were seated in the dorsal decubitus position on a regular examining surface, with knees flexed, 90 degrees of shoulder abduction, 90 degrees of elbow flexion, and the forearm in the neutral position, as seen in figure 10. (supination and pronation, 0). The investigator balanced the scapula and anterior shoulder area over the clavicle and coracoid process with one hand while rotating the shoulder internally with the other before maximum end ROM was achieved (initial position, 0).

Glenohumeral internal rotation deficit GIRD is the variation in medial rotation between the dominant and nondominant arms was used to measure the GIRD before and after treatment for each groups A and B. It is considered as loss of internal rotation dominant of $20^{\circ}$ or more as compared with the nondominant shoulder.



Figure (2): Universal goniometer.

\section{Plyometric exercise:}

The parameter recommended (1) Repetitions are from 5 to 10 repetitions for each set; (2) Sets are 1 set per motion for each exercise; (3) Frequency is 3 sessions per week; (4) Rest intervals are 60 seconds rest between each 2 exercises and 48 hours between each 2 sessions; (5) Duration is 4-6 weeks; (6) Type is multi to isolated joints; (7) Progression is $5-10 \%$ per week.

Each patient of group A were enrolled in the following sequence of plyometric; (1) chest press and throw from both sides: standing; (2) catch and throw in the air on both sides; (3) above head catch and throw (hand-to-hand); (4) unilateral plyometric shoulder exercises using elastic resistance target internal rotator; (5) bouncing a rounded ball: aim internal rotator while prone; (6) unilateral side catch and throw target internal rotator.

\section{Data Analysis:}

Descriptive statistics and unpaired t-test were conducted for comparison of age between groups. Normal distribution of data was checked using the Shapiro-Wilk test. Levene's test for homogeneity of variances was conducted to test the homogeneity between groups. Mann-Whitney test was conducted for comparison of WBFS between groups.

Unpaired t-test was conducted to compare the mean values of GIRD between groups. Paired t-test was conducted for comparison between pre- and post-treatment in each group. The level of significance for all statistical tests was set at $\mathrm{p}<0.05$. All statistical analysis was conducted through the statistical package for the social sciences (SPSS) version 25 for windows (IBM SPSS, Chicago, IL, USA).

\section{RESULTS}

There was no significant difference between groups in age (Table 1 and figure 3 ) and WBFS (Table 2).

Table (1): Basic characteristics of participants

\begin{tabular}{|l|c|c|c|}
\hline & $\begin{array}{l}\text { Study group } \\
\text { mean } \pm \text { (SD) }\end{array}$ & $\begin{array}{c}\text { Control group } \\
\text { mean } \pm \text { (SD) }\end{array}$ & p-value \\
\hline $\begin{array}{l}\text { Age } \\
\text { (years) }\end{array}$ & $16.06 \pm 1.03$ & $16.2 \pm 1.08$ & 0.73 \\
\hline
\end{tabular}

$\mathrm{SD}$, standard deviation

Table (2): Comparison of median values of WBFS between the group $A$ and $B$

\begin{tabular}{|c|c|c|c|}
\hline & Group A & Group B & $\begin{array}{c}\text { P } \\
\text { value }\end{array}$ \\
\cline { 2 - 4 } WBedian & Median & 4 & 1 \\
\hline
\end{tabular}

\section{Effect of treatment on IR and GIRD: \\ Within group comparison:}

There was a significant decrease in GIRD post treatment compared with that of pretreatment in the group A and B (Table 3).

\section{Between groups comparison:}

There was no significant difference GIRD between both groups pre-treatment. There was a significant a significant decrease in GIRD of the group A compared with that of group B post treatment (Table 3 and figure 4).

Table (3): Mean GIRD pre- and post-treatment of the group $A$ and $B$

\begin{tabular}{|l|c|c|c|}
\hline & $\begin{array}{c}\text { Group A } \\
\text { Mean } \pm \text { SD }\end{array}$ & $\begin{array}{c}\text { Group B } \\
\text { Mean } \pm \text { SD }\end{array}$ & p value \\
\hline GIRD & & & \\
\hline Pre treatment & $24.53 \pm 2$ & $24.66 \pm 1.75$ & 0.84 \\
\hline Post treatment & $16.93 \pm 1.9$ & $20.6 \pm 1.18$ & 0.001 \\
\hline \% of change & 30.98 & 16.46 & \\
\hline & $\boldsymbol{p}=\mathbf{0 . 0 0 1}$ & $\boldsymbol{p}=\mathbf{0 . 0 0 1}$ & \\
\hline
\end{tabular}

$\mathrm{SD}$, standard deviation 


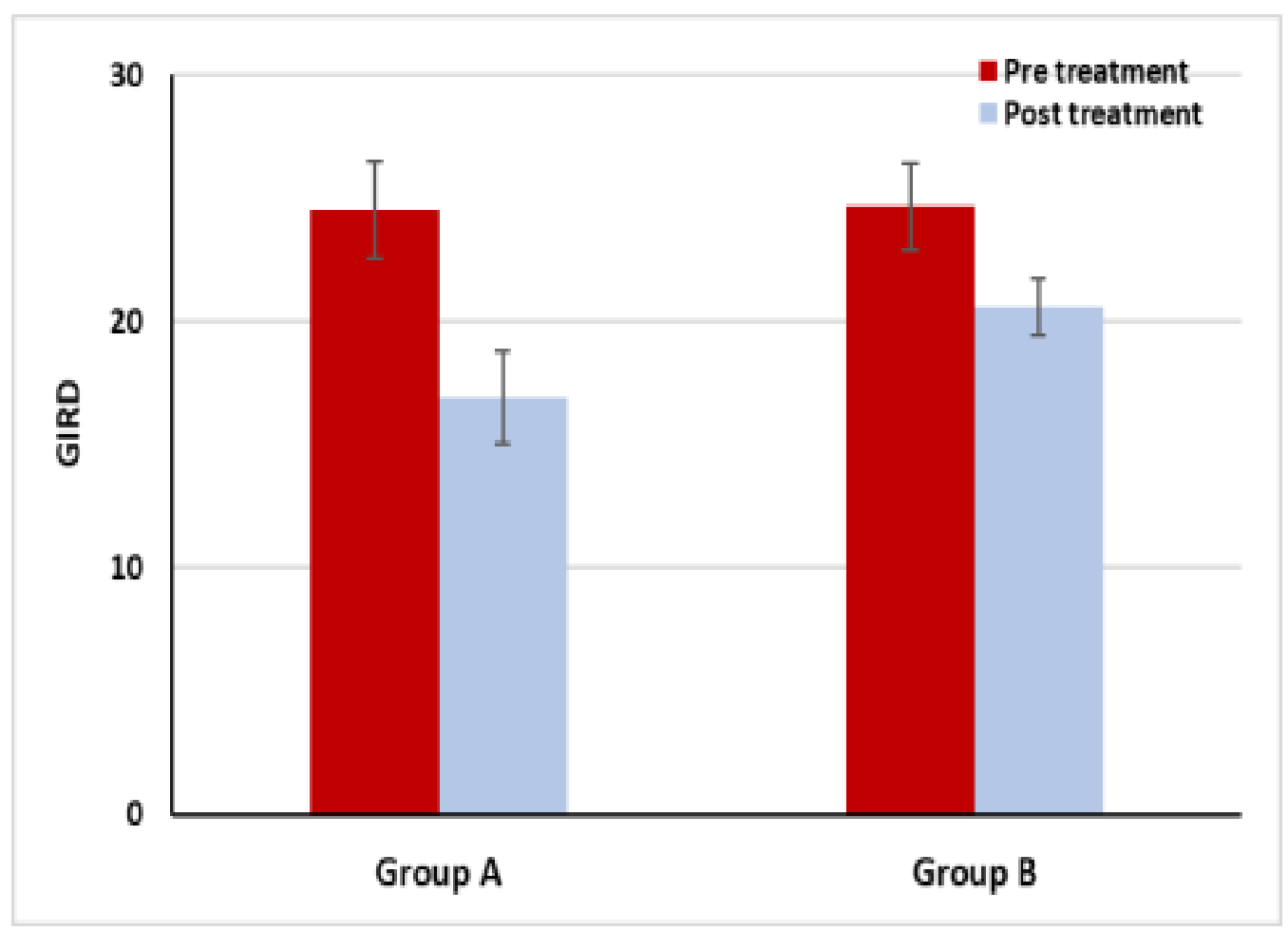

Figure (4): Comparison of GIRD between groups.

\section{DISCUSSION}

Shoulder overuse injuries are described as those that grow progressively over time and are caused by a process of excessive stress and cumulative trauma. Such accidents normally do not have a particular onset event, but rather develop over time as a result of continued physical activity, particularly where there is inadequate recovery period between episodes of physical activity [10, 11].

The current research was carried out to investigate the influence of plyometric exercise on improvement shoulder function after overuse injuries for adolescents. For these purposes, thirty boys with shoulder pain after overuse injuries with limited shoulder internal rotation were chosen. The selection of study sample agreed with Carson and Gasser ${ }^{(12)}$ who reported that the estimated age of onset is 14 years, and patients normally complain of lateral shoulder pain while throwing or doing overhead activities.

All boys participated in this study complained of shoulder pain and this agreed with Oliveira et al. ${ }^{(13)}$ who reported that older adolescent had high prevalence in shoulder pain and the involvement of pain decreased upper extremity function scores and increases deficits in internal rotation range of motion. All subjects selected in this study had marked differences in shoulder lateral and medial rotation and this agreed with Stickley et al. ${ }^{(14)}$ who reported that discrepancies in external and internal rotations in dominant shoulder are main risk factor for rotator cuff injuries for adolescents.
The degree of pain for all selected subjects was four (hurts little more) on Wong Baker faces pain scale. Garra, et al. ${ }^{(15)}$ reported that the WBF scale is used to determine level of pain for adolescents and school aged children as they are able to determine pain from fear when completing pain severity scales. All subjects in this study had been measured with universal goniometer to shoulder external and internal rotation as Donatelli et al. ${ }^{(16)}$ reported that compared to the nondominant shoulder, the dominant shoulder has less internal rotation.

All subjects selected in this study demonstrated internal rotation deficit (GIRD) compared with nondominant one, this is in parallel with Johnson et al. ${ }^{(17)}$ in their systematic review and metanalysis as they demonstrated that GIRD is widely found in young age like adolescents especially who participated in overhead activities.

The selection of plyometric exercise is compatible with Asadi ${ }^{(18)}$ as he said that in both pubertal and prepubertal populations, plyometric exercises provide the required stimulus and can improve explosive contractions. Cools et al. ${ }^{(6)}$ reported that plyometric exercises are now recommended not only in the sportspecific phase of the recovery period, but also from the onset.

From current study, we notice that plyometric exercise plays major role in injury prevention and decrease rate of shoulder and also upper extremity injuries as Swanik et al. ${ }^{(19)}$ reported that upperextremity plyometrics and strength training are used to reduce typical upper-extremity injury risk factors and improve upper-extremity efficiency. The management of these patients necessitates a detailed knowledge of 
the core concepts of dynamic stabilization reconstruction. Rehabilitation should be carried out in a step-by-step way. Range of motion, soft tissue stability, power, equilibrium, and dynamic stability of the rotator cuff and scapula can all be stressed in injury prevention and recovery services.

\section{CONCLUSION}

From obtained results of this study and the relevant literatures, it can be concluded that selected plyometric exercise added to selected physical therapy protocol has significant improvement on shoulder internal rotation after overuse injuries for adolescent who are participating in overhead activities.

\section{Financial support and sponsorship: Nil.}

\section{Conflict of interest: Nil.}

\section{REFERENCE}

1. Clarsen B, Myklebust G, Bahr R (2012): Development and validation of a new method for the registration of overuse injuries in sports injury epidemiology: the Oslo Sports Trauma Research Centre (OSTRC) Overuse Injury Questionnaire. British Journal of Sports Medicine, 47(8):495-502.

2. Bruce J, Andrews J (2014): Ulnar collateral ligament injuries in the throwing Athlete. Journal of the American Academy of Orthopaedic Surgeons, 22(5):315-325.

3. Dalton S (1992): Overuse injuries in adolescent athletes. Sports Medicine, 13(1):58-70.

4. Dubravcic-Simunjak S, Pecina M, Kuipers $\mathrm{H}$ et al. (2003): The incidence of injuries in elite junior figure skaters. The American Journal of Sports Medicine, 31(4):511-517.

5. Cools A, Palmans T, Johansson F et al. (2014): Agerelated, sport-specific adaptions of the shoulder girdle in elite adolescent tennis players. Journal of Athletic Training, 49(5):647-653.

6. Cools A, Declercq G, Cagnie B et al. (2008): Internal impingement in the tennis player: rehabilitation guidelines. British Journal of Sports Medicine, 42(3):165-171.
7. Wilk K, Meister K, Andrews J (2002): current concepts in the rehabilitation of the overhead throwing athlete. The American Journal of Sports Medicine, 30(1):136-151.

8. Yildiz Y, Aydin T, Sekir U et al. (2006): Shoulder terminal range eccentric antagonist/concentric agonist strength ratios in overhead athletes. Scandinavian Journal of Medicine and Science in Sports, 16(3):174180.

9. Bushnell B, Creighton R, Herring M (2008): Bony instability of the shoulder. Arthroscopy: The Journal of Arthroscopic \& Related Surgery, 24(9):1061-1073.

10. Arnheim D, Prentice W, Ingersoll C (1993): Principles of athletic training. Medicine \& Science in Sports \& Exercise, 25(11):1301.

11. Rauk R, Umphred D (1996): Neurological rehabilitation. Neurology Report, 64: 326-331.

12. Carson W, Gasser $\mathbf{S}$ (1998): Little leaguer's shoulder. The American Journal of Sports Medicine, 26(4):575580 .

13. Oliveira V, Pitangui A, Gomes M et al. (2017): Shoulder pain in adolescent athletes: prevalence, associated factors and its influence on upper limb function. Brazilian Journal of Physical Therapy, 21(2):107-113.

14. Stickley C, Hetzler R, Freemyer B et al. (2008): Isokinetic peak torque ratios and shoulder injury history in adolescent female volleyball athletes. Journal of Athletic Training, 43(6):571-577.

15. Garra G, Singer A, Domingo A et al. (2013): The Wong-Baker pain faces scale measures pain, not fear. Pediatric Emergency Care, 29(1):17-20.

16. Donatelli R, Ellenbecker T, Ekedahl S et al. (2000): Assessment of shoulder strength in professional baseball pitchers. Journal of Orthopaedic \& Sports Physical Therapy, 30(9):544-551.

17. Johnson J, Fullmer J, Nielsen C et al. (2018): Glenohumeral internal rotation deficit and injuries: A systematic review and meta-analysis. Orthopaedic Journal of Sports Medicine, 6(5):2325-2328.

18. Asadi A (2013): Effects of in-season short-term plyometric training on jumping and agility performance of basketball players. Sport Sciences for Health, 9(3):133-137.

19. Swanik K, Thomas S, Struminger A et al. (2016): The effect of shoulder plyometric training on amortization time and upper-extremity kinematics. Journal of Sport Rehabilitation, 25(4):315-323. 